Article

\title{
Temperature and Field Induced Variations of Electric Conductivities of HTV Silicone Rubbers Derived from Measured Currents and Surface Potential Decay Characteristics
}

\author{
Shahid Alam, Yuriy V. Serdyuk *(1) and Stanislaw M. Gubanski \\ Department of Electrical Engineering, Chalmers University of Technology, 41296 Gothenburg, Sweden; \\ shahcth@gmail.com (S.A.); stanislaw.gubanski@chalmers.se (S.M.G.) \\ * Correspondence: yuriy.serdyuk@chalmers.se
}

Received: 26 April 2020; Accepted: 8 June 2020; Published: 10 June 2020

\begin{abstract}
The temperature and field dependencies of electric conductivities of two types of silicone rubber-based polymers intended for use in high voltage direct current applications are presented and discussed. The conductivities obtained with the standard method by measuring a current through the material sample placed between metallic electrodes in response to the applied voltage are compared with those deduced from the measured potential decay on pre-charged material surface in an open circuit configuration. The measurements were conducted in the range of the applied electric field strength (0.5-5) $\mathrm{kV} / \mathrm{mm}$ and temperatures ranging from $22^{\circ} \mathrm{C}$ to $70^{\circ} \mathrm{C}$. It is shown that the values of the conductivities obtained by the two methods are in agreement and their temperature dependences obey Arrhenius law yielding similar activation energies.
\end{abstract}

Keywords: silicone rubber; electric conductivity; charging current; surface potential decay; activation energy

\section{Introduction}

High voltage direct current (HVDC) electric energy transmission is the key technology for transporting bulk power for long distances, linking remotely located renewable energy sources (e.g., offshore wind parks) to mainland, interconnecting and balancing alternating current (AC) grids. The implementation of HVDC technology demands reliable and smooth operation of insulation systems of the components that is determined by the performance and properties of constituting insulating materials. Nowadays, composites based on silicone rubbers become increasingly popular in HVDC insulation due to their high electric withstand performance, lower weight compared to traditionally used porcelain and glass, elasticity, etc. In addition, they provide hydrophobic properties vital for impeding surface leakage currents and thus for enhancing the withstand ability against surface flashovers [1]. To enhance their mechanical and electrical properties and to meet specific requirements for outdoor insulation (e.g., arc endurance), various fillers, such as fumed silica, quartz and alumina try-hydrate (ATH), are added to the base material $[2,3]$. Due to the presence of fillers, the composites may exhibit non-linearities in their electrical characteristics, specifically in the electric conductivity, when exposed to strong unipolar HVDC stresses, as well as elevated temperatures, which are normal operating conditions, e.g., for converter transformer bushings, HVDC cables terminations, etc. The non-linear conductivities reflect the effects of electric charges in the material bulk, which may be injected from metallic elements and accumulated in the insulation; in particular, on internal filler-base material interfaces. Since the electric conductivity plays a dominant role in controlling 
electric field distributions in HVDC insulation systems, its accurate determination is essential for the proper selection of materials and designing insulation elements.

Measurements of electric conductivities of highly resistive materials are usually performed utilizing a standardized approach [4,5], where a material sample is placed between energized electrodes and a current through it is recorded during a certain time interval. The direct current (DC) conductivity is obtained from the magnitude of the current at a steady state reaching, which requires measuring time ranging from tens of hours to a few days (depending on material nature) to mitigate slow dielectric polarization processes [6,7]. Moreover, despite the simplicity of the method, its implementation for insulating materials is not straightforward, due to the necessity of registering currents on sub-picoamperes levels, or even lower. In addition, structural features of test samples, ambient conditions in the measurement cell, processes at metal-material interfaces, etc., may affect the outcome of the experiments [8-10]. A summary of the effects of such factors is presented in [7].

The influence of the electrodes of the test cell on experimental results requires special consideration, since it may become significant, especially at elevated temperatures, when specific processes in the material and on metal-material interfaces are activated. These include, e.g., field assisted charge injection at the interfaces and excessive mechanical stresses due to thermal expansions, especially in cases of soft material samples. The former may lead to situations when the intrinsic conductivity cannot be achieved due to the presence of external charges in the material, whereas the latter may give rise to erroneous results due to the induced deformations of the samples. Such effects can be avoided by using non-contact techniques, and attempts to develop such methods have been undertaken recently. An example is the methodology for deducing volumetric conductivities from surface potential decay (SPD) characteristics measured on pre-charged materials samples in an open circuit configuration [11-13]. It has been shown in [13], where field-dependent bulk conductivities of several types of silicone rubbers were studied at room temperature, that the SPD-based technique provided materials conductivities rather close to those obtained by the standard method. Furthermore, it has been noticed that, in addition to the reduced influence of charge injection due to avoiding at least one of the two metal-material contacts, the SPD method offers a number of advantages such as a shorter measuring time needed for the evaluation of field dependencies of the conductivities, a broader range of test fields (controlled by charging conditions), no necessity of measuring extremely low currents, etc.

In the present study, the work reported in [13] on utilizing the SPD technique for determining field dependent conductivities is extended to elevated temperatures, with the aim to further examine its feasibility. Conductivities of two types of silicone rubber-based materials retrieved with the standard and SPD-based approaches for the range of the electric field strength $(0.5-5) \mathrm{kV} / \mathrm{mm}$ and temperatures (22-70) ${ }^{\circ} \mathrm{C}$ are compared. Furthermore, values of the activation energies deduced from the temperature dependences of the conductivities obtained with both methods are discussed.

\section{The Experiment}

\subsection{Materials Samples}

In the present study, flat samples, $100 \times 100 \mathrm{~mm}^{2}$, of high-temperature vulcanized silicon rubber (SIR) of type Elastosil R401/50 were used. The rubber was based on polydimethylsiloxane reinforced with silica fillers. In one case, the original material (denoted as A in Table 1), manufactured by curing with dicumylperoxide and degassed during $17 \mathrm{~h}$ at $70{ }^{\circ} \mathrm{C}$, was used. In another case, it was doped with $50 \%$ of ATH (referred to as B in Table 1). The filler was of type OL-104 ZO, a vinylsilane treated finely precipitated aluminium hydroxide, which is commonly used to enhance arc resistance of SIR-based composites. The dielectric constants $\varepsilon_{r}$ and surface conductivities $K_{\mathrm{s}}$ of the materials were determined using the Insulation Diagnostic System IDAX300 and Keithley 6517A electrometer, respectively. For both types of measurements, the resistivity test fixture Keithley 8009 was used. The results are shown in Table 1, together with the thicknesses of the materials samples evaluated, as an average of four measurements taken at various distances from the edge of the sample. 
Table 1. Specification of the studied materials, measured surface conductivities $K_{s}($ at $1 \mathrm{kV})$, dielectric constants $\varepsilon_{r}$ (at $50 \mathrm{~Hz}$ ) and thickness of the samples.

\begin{tabular}{ccccccc}
\hline Material & $\begin{array}{c}\text { Commercial } \\
\text { Name }\end{array}$ & $\begin{array}{c}\text { Curing } \\
\text { Agent }\end{array}$ & $\begin{array}{c}\text { Additional } \\
\text { Filler }\end{array}$ & $\boldsymbol{K}_{s} \mathbf{1 0}^{-\mathbf{1 7}}, \mathbf{S}$ & $\varepsilon_{r}$ & $\begin{array}{c}\text { Thickness, } \\
\mathbf{m m}\end{array}$ \\
\hline A & $\begin{array}{c}\text { Elastosil } \\
\text { R401/50 }\end{array}$ & peroxide & - & $4.5 \pm 0.9$ & $2.4 \pm 0.03$ & $0.256 \pm 0.024$ \\
\hline B & $\begin{array}{c}\text { Elastosil } \\
\text { R401/50 }\end{array}$ & peroxide & $50 \%$ ATH & $10 \pm 1.1$ & $3.5 \pm 0.05$ & $0.360 \pm 0.034$ \\
\hline
\end{tabular}

\subsection{Setup and Procedure for Measuring Volume Currents}

The measurements of the volumetric electric conductivities were performed following the ASTM standard D257. Like in the measurements of the surface conductivities mentioned above, a material sample was placed between the electrodes in the resistivity test fixture, where a three-electrode arrangement, consisting of a measuring, grounded and guard electrodes was implemented. The test cell was connected to the electrometer, which was used to energize the sample by applying a test voltage and for recording the resulted current through the material. The maximum test voltage was $1 \mathrm{kV}$ DC and the device provided a measurement range for the current from $1 \mathrm{fA}$ to $20 \mathrm{~mA}$. Further details about the set-up can be found elsewhere in [7].

The test procedure was based on applying the test voltage in a step-wise manner for obtaining a dependence of the conductivity within a range of the electrical field strength. Thus, on the first step, a DC voltage of $300 \mathrm{~V}$ was applied, and on the following steps it was increased to $600 \mathrm{~V}, 800 \mathrm{~V}, 900 \mathrm{~V}$ and $1 \mathrm{kV}$. For each stress level, the resulting bulk current was recorded. It was observed that each rapid increase of the voltage resulted in a spike of the capacitive current, which monotonously decayed during rather long time (due to slow polarization processes in the material), and finally, the total current reached its steady state. The magnitude of the steady current was recorded and was utilized for deducing material conductivity.

For conducting the measurements at elevated temperatures, the test fixture with the sample was placed inside an oven (Memmert Universal UN 55) that provided controlled isothermal conditions. The same measurement procedure was followed as for the room temperature. Each measurement was repeated at least three times under similar conditions to check the repeatability of the results. The samples were kept short-circuited and grounded between the consecutive measurements.

\subsection{Setup and Procedure for SPD Measurements}

The experimental setup, including arrangements for charging material sample surface by corona and for monitoring the induced surface potential, was built inside a sealed metallic chamber. The internal volume was $\sim 1 \mathrm{~m}^{3}$. As seen in the photograph of the assembled components of the setup in Figure 1, it included a linear positioning system carrying a grounded metallic table. For measurements at room temperature, the material sample was placed directly on the table, whereas at elevated temperatures, it was placed on a hot plate with the dimensions $140 \mathrm{~mm} \times 140 \mathrm{~mm}$ (PZ 35 ET, Harry Gestigkeit $\mathrm{GmbH}$ ) mounted on the table. The hot plate was energized from a control unit via a low voltage busing. The temperature of the plate was first adjusted to the desired value and the material sample was left on it for $30 \mathrm{~min}$ for achieving steady state thermal conditions. Thereafter, corona charging and SPD measurements were performed as described below.

To deposit charges on the material surface, corona discharge from a needle (tip radius $125 \mu \mathrm{m}$ ) was utilized. During corona charging, the needle was positioned against the center of the sample normal to its surface and the tip was at $2 \mathrm{~mm}$ from it. The needle was energized from an external DC high voltage supply connected to it via HV bushing (not shown in Figure 1). The duration of the charging phase was $2 \mathrm{~min}$ and it was performed at ambient pressure. After completing the charging, the corona was terminated and the pressure in the chamber was reduced to $300 \pm 10$ mbar, to minimize the neutralization of the deposited charges by air ions. Afterwards, the table with the 
sample was moved beneath the probe fixed on the same wooden arm and the profile of the surface potential vs. was recorded. The measurements were repeated with certain time interval. The potential decay characteristics were obtained from the recorded distributions at the location on the surface corresponding to the maximum vs. value. The potential was measured by means of a Kelvin-type vibrating probe (Trek 3455ET) and electrostatic voltmeter (Trek 341B, $\pm 20 \mathrm{kV}$ ). A detailed description of the procedure can be found in [7].

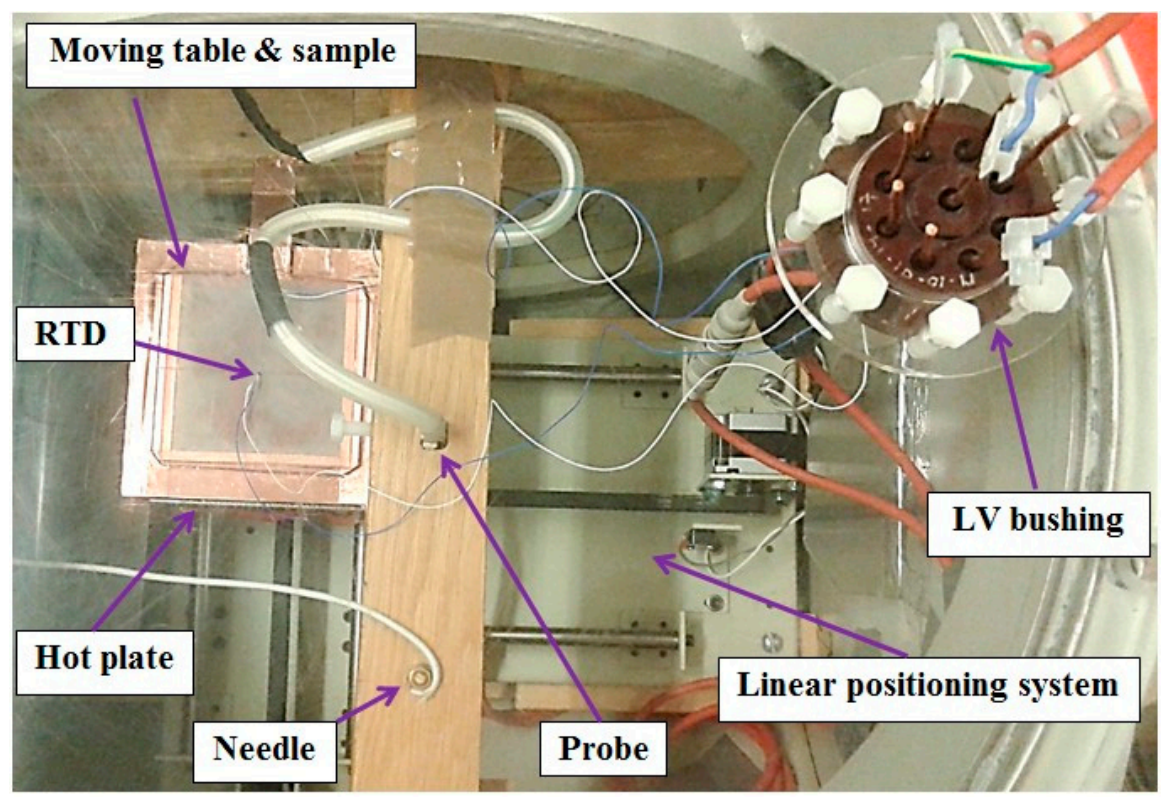

Figure 1. Top view of the set-up for surface potential decay (SPD) measurements. The movable table with the material sample is intentionally shifted to the left from its normal position for visibility. The table motion is in $x-y$ plane (horizontally and vertically in the picture plane) and its motion and position are programmed from the external control unit. The corona needle and the potential probe are mounted on the wooden arm and are directed downwards to the sample (therefore, not visible on the photograph). RTD indicates position of the temperature sensor during the analysis of thermal conditions (was removed during SPD measurement).

It is worth mentioning that before the SPD measurements at elevated temperatures were taken, the uniformity of thermal conditions in the sample was examined. Two resistive temperature detectors (RTD) were installed in the middle of the sample surface and at its edge. They were connected to a multimeter (Fluke $88464 \mathrm{~A}$ ) via bushing. The surface temperatures were recorded at different temperatures of the hot plate and at reduced pressure inside the chamber. The results of the measurements are provided in Table 2 and indicate that the temperature gradients through the thickness of the samples were insignificant and, therefore, their possible impact could be neglected.

Table 2. Measured temperatures on material sample at different temperatures of the hot plate. The mean values and standard deviations are obtained from five different measurements.

\begin{tabular}{ccccc}
\hline Surface & \multicolumn{4}{c}{ Hot Plate Temperature, ${ }^{\circ} \mathbf{C}$} \\
\cline { 2 - 5 } Temperature, ${ }^{\circ} \mathbf{C}$ & $\mathbf{4 0}$ & $\mathbf{6 0}$ & $\mathbf{7 0}$ & $\mathbf{8 0}$ \\
\hline$R T D_{\text {mid }}$ & $39.8 \pm 0.06$ & $59.6 \pm 0.12$ & $69.0 \pm 0.21$ & $78.3 \pm 0.36$ \\
$R T D_{\text {edge }}$ & $39.2 \pm 0.08$ & $58.8 \pm 0.17$ & $67.9 \pm 0.27$ & $76.8 \pm 0.45$ \\
\hline
\end{tabular}

To conclude this section, it is important to emphasize that the extremely low surface conductivities $K_{\mathrm{s}}$ of the studied materials (recall data in Table 1 ) and the procedure for conducting SPD measurements at reduced gas pressure prevented contributions of the leakage of pre-deposited charges through the 
sample surface and their neutralization by air ions, respectively, that ensured that conduction through material bulk was the main cause of the SPD in the present study.

\section{Results and Discussion}

\subsection{Volume Currents}

The recorded volume currents are shown in Figure 2. One may notice pronounced spikes of the capacitive currents which appeared at each increase of the test voltage. The amplitudes of these current spikes were higher at higher temperatures as the base level of the current increases. It can be observed that the decaying polarization current is strongly influenced by the material composition, electrical field strength and temperature. Thus, times needed to reach steady volume currents are different for the studied materials. Quantitatively, the relaxation time after applying the first voltage step is almost twice as short for the ATH doped silicone rubber B at room temperature as the relatively pure material A. These can be attributed to the variations in materials compositions and their structural features (a discussion in this regard can be found in [7]). The increase of temperature accelerated the polarization processes in both materials and thus the time to (quasi-) steady state is much shorter at $70{ }^{\circ} \mathrm{C}$ than at room temperature. Nevertheless, a decay of the polarization current is noticeable for silicone rubber A, even at the maximum studied temperature of $70{ }^{\circ} \mathrm{C}$. For ATH doped material B, the current decay is much faster under the same conditions. The magnitudes of the steady currents are significantly higher at elevated temperatures.

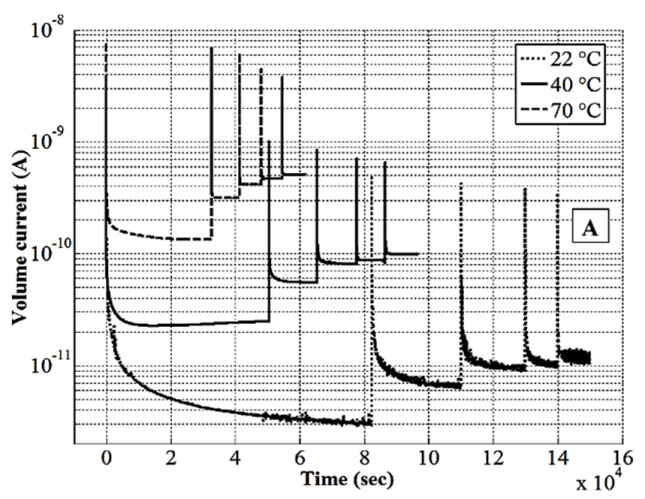

(a)

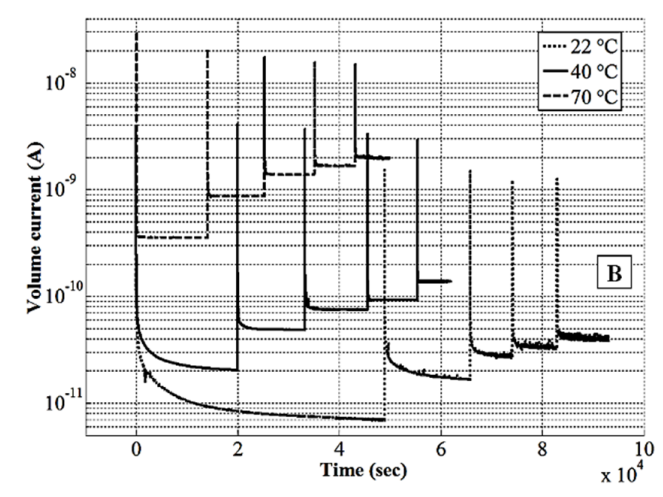

(b)

Figure 2. Recorded volume currents through samples of material A (a) and alumina try-hydrate (ATH) doped material B (b) at different temperatures. Recall that the test voltage is applied in increasing steps (from left to right on the time axis).

As mentioned in the previous section, combined electrical and thermal stresses may cause injection and space charge accumulation in the material $[14,15]$. To examine the strength of these effects in the present experiments, the approach outlined in [13] was used and the densities $J$ of the measured currents are plotted as functions of the applied electrical field $E$ in $\log -\log$ coordinates in Figure 3 (note that the line with the slope equal to unity in such a plot indicates pure Ohmic behavior [16]). As seen, the $J(E)$ characteristics for silicone rubber A, obtained by utilizing the quasi steady-state currents from Figure 2, are close to the line with the unit slope and slight deviations are observed at higher fields and temperatures. For material B, the deviations appear at lower field levels and lower temperatures compared to material A. One should stress, however, that the deviations from Ohmic behavior are fairly weak for both materials, indicating that the threshold fields, above which the injected and accumulated space charges may become significant, were not reached, even at the elevated temperatures. Therefore, the currents recorded in both materials are due to the intrinsic conduction and the respective conductivities can be considered as being close to true material characteristics under the conditions of the present study. 


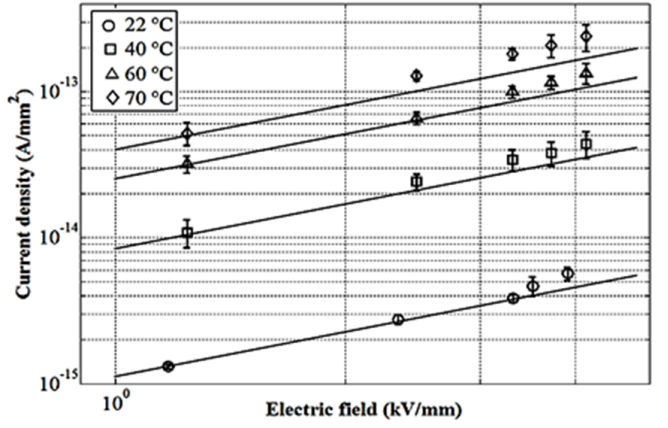

(a)

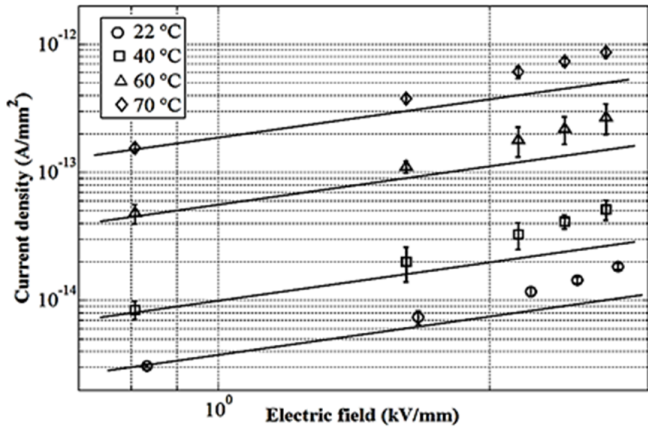

(b)

Figure 3. Field strength—current density characteristics obtained for materials A (a) and B (b) at different temperatures. The lines indicate the unit-slopes, the error bars show the standard deviations.

\subsection{Surface Potential Decay}

Normalized SPD characteristics obtained at different temperatures are demonstrated in Figure 4. As seen, the temperature strongly accelerates surface potentials decay (note the significant shift of the curves to the left). For silicone rubber A, the time needed for the potential to drop down to the half of its initial value is approximately $5200 \mathrm{~s}$ at $22{ }^{\circ} \mathrm{C}$ and it is reduced to $1750 \mathrm{~s}, 700 \mathrm{~s}$ and $420 \mathrm{~s}$ at temperatures $40^{\circ} \mathrm{C}, 60^{\circ} \mathrm{C}$ and $70^{\circ} \mathrm{C}$, respectively. For material B, the respective times are shorter-approximately $1120 \mathrm{~s}, 450 \mathrm{~s}, 290 \mathrm{~s}$ and $230 \mathrm{~s}$. Thus, the total reduction due to the temperature rise is about 12 and 5 times for materials A and B, respectively. Based on this observation, one can conclude that the variations of the conductive properties induced by the temperature and the electric field in the bulk (which decreases with time due to the decaying surface potentials) of rubber A are more pronounced than for the material B. At the same time, the decay in material B is faster as compared to material A; this may be due the to the presence of a large amount of ATH filler.

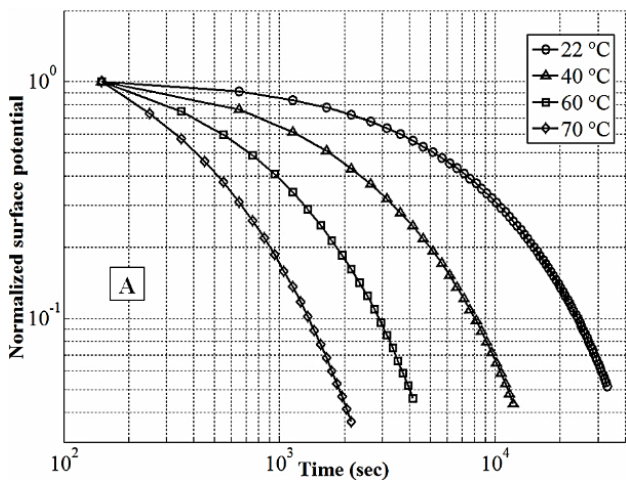

(a)

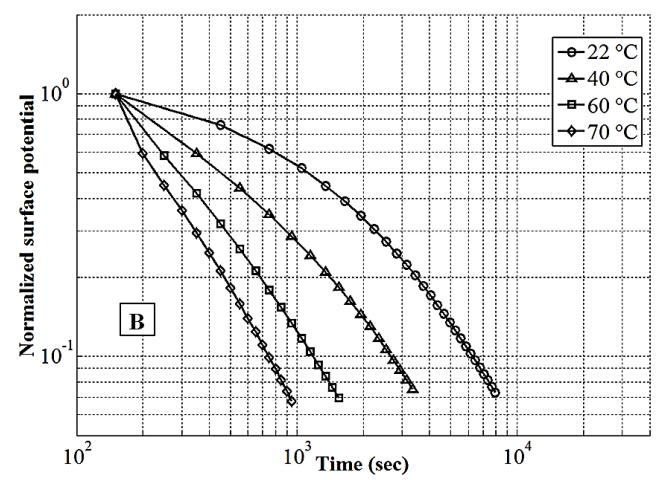

(b)

Figure 4. Normalized surface potential decay measured for materials A (a) and B (b) at different temperatures. Note that the initial potential (right after charging) is used as a reference; the temperature is for the hot plate.

\subsection{Bulk Conductivities Deduced from SPD Characteristics}

As mentioned in Section 2.3, the volume conduction is the dominating mechanism of surface potential (and therefore surface charge) decay in the present study. Under this condition, the material bulk conductivity can be deduced from the SPD rate as [17];

$$
\mathrm{K}_{\mathrm{v}}=-\varepsilon_{0} \varepsilon_{r} \frac{1}{\mathrm{~V}_{\mathrm{s}}(\mathrm{t})} \frac{\mathrm{dV} \mathrm{s}(\mathrm{t})}{\mathrm{dt}}
$$


where $\varepsilon_{0}$ stands for the permittivity of vacuum, and $t$ is time. The derivation of (1), based on the analysis of the decay of charges on gas-solid interfaces due to different mechanisms (bulk processes, gas neutralization and surface conduction), can be found elsewhere in [7]. Note that, in contrast to the standard method, the field dependence of the conductivity $K_{\mathrm{v}}(E)$ is derived from a single SPD characteristic, since the field $E$ in the bulk decays naturally as $E(t) \sim$ vs. $(t)$ and its magnitude is found as the ration of the potential and the thickness of the sample.

The SPD rates obtained from the numerical differentiation of the measured SPD characteristics and the values of the dielectric constants from Table 1 were used to calculate $K_{\mathrm{v}}$ according to (1). The obtained field dependences $K_{\mathrm{v}}(E)$ for materials $\mathrm{A}$ and $\mathrm{B}$ at different temperatures are shown in Figure 5, together with the results of the volume current-based measurements. Note that curves indicating data points received from both methods at the same temperature are marked by the encircled areas. As can be observed, the field dependencies of the conductivities of both materials are rather weak in the studied range of the field strength, but the temperature variations are strong and non-linear. In general, the values of $K_{\mathrm{v}}$ vary within two orders of magnitudes for the studied temperature range. Both methods provided results demonstrating the same trends; however, the discrepancies between the obtained conductivity values are different for the studied materials and may be rather high, as can be seen in Table 3. Thus, the volume currents in general yielded a higher conductivity of material A compared to that obtained with the SPD method, except at room temperature, where the opposite behavior is observed and the results are close. At elevated temperatures, the discrepancies are rather stable in the entire studied range of the applied field. For material B, the best agreement was achieved at $40^{\circ} \mathrm{C}$, and the deviations between the results from the two methods are higher at room temperature as well as at higher temperatures, despite the different trends (note different signs at the percentage deviations). A possible cause of such behaviour may be attributed to a thermal expansion of the materials samples at elevated temperatures. Placed between the electrodes in the resistivity test fixture during the current measurement, the sample might experience a constant pressure and, since the studied materials are soft, sample thickness could decrease slightly, due to a thermal expansion causing stronger applied field strength and, respectively, larger volume currents. In contrast, when using the SPD technique, deformations of the samples are avoided.

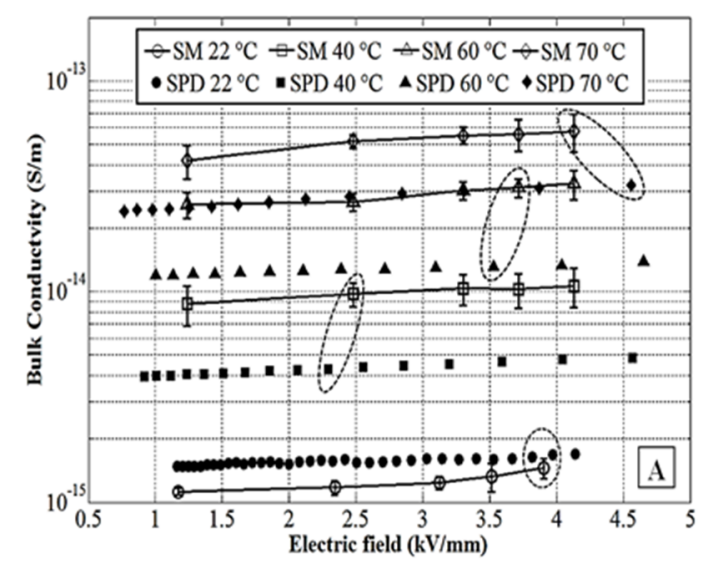

(a)

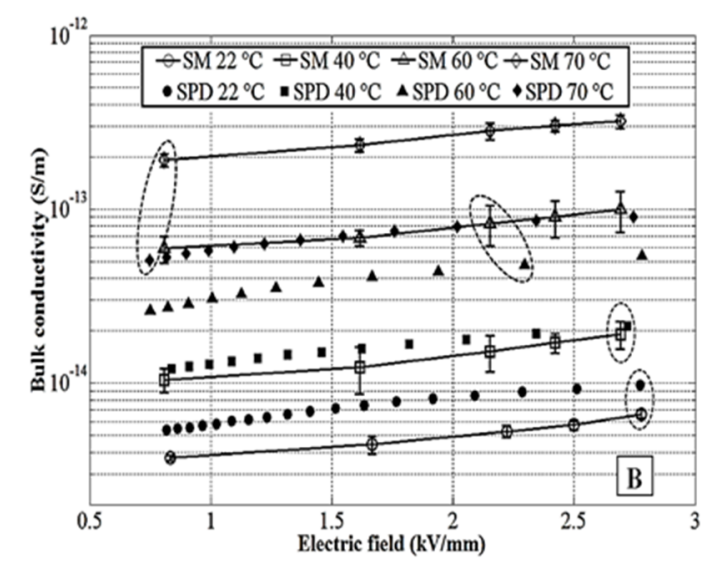

(b)

Figure 5. Field dependencies of the bulk conductivities of materials A (a) and B (b) at different temperatures obtained from the SPD characteristics and standard measurements (SM in the legends). 
Table 3. Deviations of $K_{v}$ values for materials A and B (shown as the ration A/B), obtained from the SPD characteristics at different temperatures, as compared to the standard measurements.

\begin{tabular}{cccccc}
\hline \multirow{2}{*}{ Temperature, ${ }^{\circ} \mathbf{C}$} & \multicolumn{5}{c}{ Deviations, \% } \\
\cline { 2 - 6 } & $\mathbf{3 0 0 V}$ & $\mathbf{6 0 0 V}$ & $\mathbf{8 0 0 V}$ & $\mathbf{9 0 0 V}$ & $\mathbf{1 ~ k V}$ \\
\hline 22 & $-31 /-44$ & $-33 /-67$ & $-30 /-69$ & $-21 /-55$ & $-13 /-47$ \\
40 & $54 /-15$ & $55 /-27$ & $56 /-17$ & $54 /-13$ & $55 /-12$ \\
60 & $53 / 54$ & $54 / 40$ & $57 / 47$ & $58 / 47$ & $59 / 46$ \\
70 & $40 / 72$ & $45 / 70$ & $46 / 72$ & $45 / 72$ & $45 / 72$ \\
\hline
\end{tabular}

The temperature dependences of the conductivities are further analyzed using Arrhenius law:

$$
\mathrm{K}_{\mathrm{v}}(\mathrm{T})=\mathrm{K}_{\mathrm{v} 0} \exp \left(-\frac{\mathrm{E}_{\mathrm{a}}}{\mathrm{kT}}\right)
$$

Here, $K_{v 0}$ is a constant, $k$ is Boltzmann's constant, $E_{a}$ stands for the activation energy, and $T$ is the absolute temperature. The results of the fitting of data from Figure 5 by using (2) are shown in Figure 6. One may notice that the dependences $K_{v}(T)$ obtained by both methods obey Arrhenius law. The activation energies deduced from the standard measurements are slightly higher than those from the SPD technique. Additionally, the difference in $E_{a}$ for ATH filled material B is larger than for material A. In general, however, the activation energies are close to the values reported earlier for silicone rubbers $[18,19]$.

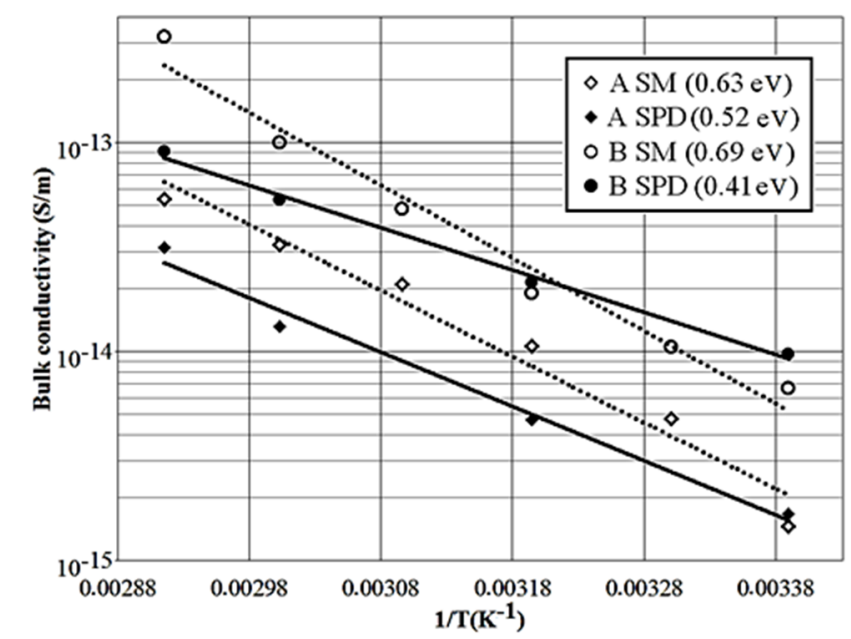

Figure 6. Bulk conductivities as functions of reciprocal of absolute temperature for pure and ATH filled materials (indicated as A and B in the legend). The slopes of the lines corresponding to activation energies are given in the brackets in the legends.

\section{Conclusions}

Temperature and field dependencies of the electric volume conductivities of two types of HTV silicone rubber (original and doped with 50\% ATH filler) have been determined by measurements of volumetric currents and surface potential decay. It is shown that materials compositions have strong impact on the measured volume currents and SPD characteristics at elevated temperatures. The conductivities obtained by different methods are found to be in a reasonable agreement and reveal the significant effect of temperature, whereas their field dependencies are rather weak. The temperature dependencies of the conductivities of both materials are of Arrhenius type and the activation energies deduced from the results obtained with the two methods are close to the levels reported in the literature. Based on the analysis of the results, the SPD-based technique can be proposed as an alternative method for the electrical characterization of insulating materials at elevated temperatures. 
Author Contributions: All the authors (S.A., Y.V.S. and S.M.G.) contributed equally to the compilation of this paper. Their contributions included suggestions for conducting measurements, the analysis of the obtained results and refining the manuscript. S.A. prepared the experimental setups and performed the measurements. All authors have read and agreed to the published version of the manuscript.

Funding: The authors acknowledge the financial support provided by ABB CR Sweden and Chalmers Area of Advance Energy.

Acknowledgments: The authors are grateful to Lars Palmqvist, Birgitta Källstrand and Olof Hjortstam (ABB CR, Västerås, Sweden) for valuable discussions and for providing material samples for the experiments.

Conflicts of Interest: The authors declare no conflict of interest.

\section{References}

1. Arshad, A.; Nekahi, A.; McMeekin, S.G.; Farzaneh, M. Flashover Characteristics of Silicone Rubber Sheets under Various Environmental Conditions. Energies 2016, 9, 683. [CrossRef]

2. Ramirez, I.; Jayaram, S.; Cherney, E.; Gauthier, M.; Simon, L. Erosion resistance and mechanical properties of silicone nanocomposite insulation. IEEE Trans. Dielectr. Electr. Insul. 2009, 16, 52-59. [CrossRef]

3. Ghunem, R.A.; Jayaram, S.H.; Cherney, E.A. The DC inclined-plane tracking and erosion test and the role of inorganic fillers in silicone rubber for DC insulation. IEEE Electr. Insul. Mag. 2014, 31, 12-21. [CrossRef]

4. International standard IEC 62631-3-1. Dielectric and Resistive Properties of Solid Insulating Materials-Part 3-1: Determination of Resistive Properties (DC Methods)-Volume Resistance and Volume Resistivity-General Method; IEC: Geneva, Switzerland, 2016.

5. ASTM D257-14. Standard Test Methods for DC Resistance or Conductance of Insulating Materials; ASTM International: West Conshohocken, PA, USA, 2014.

6. Tsekmes, I.A. Electrical Characterization of Polymeric DC Mini-Cables by Means of Space Charge \& Conduction Current Measurements. Ph.D. Thesis, Delft University of Technology, Delft, The Netherlands, 2012.

7. Alam, S. Surface Potential Dynamics on Insulating Polymers for HVDC Applications. Ph.D. Thesis, Chalmers University of Technology, Gothenburg, Sweden, 2016.

8. Rakowska, A. Influence of different test conditions on volume resistivity of polymeric insulated cables and polyethylene samples. In Proceedings of the Eighth International Conference on Dielectric Materials, Measurements and Applications, Edinburgh, UK, 17-21 September 2000; Institution of Engineering and Technology (IET): London, UK; pp. 281-284.

9. Nelson, J.K. Dielectric Polymer Nanocomposites; Springer: New York, NY, USA, 2010.

10. Viertel, J.; Petersson, L.; Friberg, A.; Dominguez, G.; Törnkvist, C. Electrode influence on dc conductivity measurements of low density polyethylene. In Proceedings of the IEEE International Conference Solid Dielectrics, Bologna, Italy, 30 June-4 July 2013; pp. 1048-1051.

11. Noras, M.A. Non-Contact Surface Charge/Voltage Measurements Fieldmeter and Voltmeter Methods; Trek Application Note, Number 3002; Trek Inc.: New York, NY, USA, 2002.

12. Lutz, B.; Kindersberger, J. Determination of volume resistivity of polymeric insulators by surface charge decay. In Proceedings of the 16th International Symposium on High Voltage Engineering 2009, Johannesburg, South Africa, 24-28 August 2009; pp. 28-33.

13. Alam, S.; Serdyuk, Y.V.; Gubanski, S.M. Field-dependent electric conductivities of silicone rubbers deduced from measured currents and surface potential decay characteristics. Int. J. Polym. Anal. Charact. 2018, 24, 54-62. [CrossRef]

14. Raju, G.G. Dielectrics in Electric Fields; Dekker Inc.: New York, NY, USA, 2003.

15. Lampert, M.A. Simplified Theory of Space-Charge-Limited Currents in an Insulator with Traps. Phys. Rev. 1956, 103, 1648-1656. [CrossRef]

16. Auge, J.; Montanari, G.; Laurent, C.; Fabiani, D. Investigating dc polyethylene threshold by space charge. Current and electroluminescence measurements. IEEE Trans. Dielectr. Electr. Insul. 2000, 7, 797-803. [CrossRef]

17. Molinié, P. Measuring and modeling transient insulator response to charging: The contribution of surface potential studies. IEEE Trans. Dielectr. Electr. Insul. 2005, 12, 939-950. [CrossRef] 
18. Nguyen, D.; Sylvestre, A.; Gonon, P.; Rowe, S. Dielectric properties analysis of silicone rubber. In Proceedings of the 2004 IEEE International Conference on Solid Dielectrics, Toulouse, France, 5-9 July 2004; Institute of Electrical and Electronics Engineers (IEEE): Toulouse, France, 2004; pp. 103-106.

19. Hillborg, H.; ABB, Västerås, Sweden. Private communication, 2016.

(C) 2020 by the authors. Licensee MDPI, Basel, Switzerland. This article is an open access article distributed under the terms and conditions of the Creative Commons Attribution (CC BY) license (http://creativecommons.org/licenses/by/4.0/). 